\title{
Evolutionary history and genetic parallelism affect correlated responses to evolution
}

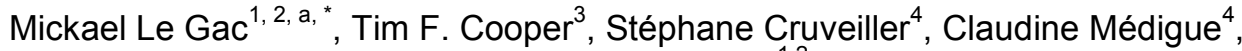 \\ Dominique Schneider ${ }^{1,2}$
}

\author{
${ }^{1}$ Laboratoire Adaptation et Pathogénie des Microorganismes, Université Joseph Fourier, Grenoble Cedex 9, \\ France \\ ${ }^{2}$ CNRS UMR 5163, Grenoble Cedex 9, France \\ ${ }^{3}$ Department of Biology and Biochemistry, University of Houston, Houston, TX, USA \\ ${ }^{4}$ CNRS-UMR 8030 \& CEA/DSV/IG/Genoscope LABGeM, Evry Cedex, France \\ ${ }^{a}$ Current address : DYNECO/Pelagos, Ifremer, Plouzané, France
}

*: Corresponding author : Mickaël Le Gac, fax: (+33) 298224548 ; email address : mickael.le.gac@ifremer.fr

\begin{abstract}
:
We investigated the relationship between genomic and phenotypic evolution among replicate populations of Escherichia coli evolved for 1000 generations in four different environments. By resequencing evolved genomes, we identified parallel changes in genes encoding transcription regulators within and between environments. Depending on both the environment and the altered gene, genetic parallelism at the gene level involved mutations that affected identical codons, protein domains or were widely distributed across the gene. Evolved clones were characterized by parallel phenotypic changes in their respective evolution environments but also in the three alternative environments. Phenotypic parallelism was high for clones that evolved in the same environment, even in the absence of genetic parallelism. By contrast, clones that evolved in different environments revealed a higher parallelism in correlated responses when they shared mutated genes. Altogether, this work shows that after an environmental change or the colonization of a new habitat, similar ecological performance might be expected from individuals that share mutated genes or that experienced similar past selective pressures.
\end{abstract}

Keywords : adaptation ; Epistasis ; experimental evolution ; genome sequencing ; parallelism 


\section{Introduction}

Next-generation sequencing (NGS) technologies are revolutionizing our understanding of the genetics of adaptation. From evolution studies focusing on specific candidate genes, investigations have now switched to the genome scale. Applied to experimental evolution of microorganisms, genome comparisons of ancestral and evolved clones enable the identification of virtually all the mutations associated with experimental adaptation (reviewed in Brockhurst et al. 2011; Conrad et al. 2011; Dettman et al. 2012; Hindré et al. 2012). A prominent finding has been that adaptation of independent replicate populations to a given environment often involves genetic parallelism, characterized by mutations that affect identical genes, operons, or functional operational units (Herring et al. 2006; Barrick et al. 2009; Conrad et al. 2009; Tenaillon et al. 2012). However, the precise mutations altering these genetic loci are often different in replicate bacterial populations (Woods et al. 2006; Tenaillon et al. 2012).

Consideration of the genes that are repeatedly mutated during evolution experiments finds that many are global regulators of gene expression, highlighting the involvement of regulatory network rewiring in adaptation (Philippe et al. 2007; Wang et al. 2010; Conrad et al. 2011; Hindré et al. 2012). The same global regulator-encoding genes are often mutated in distinct experimental settings indicating that identical regulatory hubs may provide adaptation to contrasting environments (Conrad et al. 2011). Moreover, non-additive, i.e. epistatic, interactions between mutations have been shown to be of primary importance in explaining both the evolutionary trajectory of a population and the phenotypes of evolved individuals (Bull et al. 2000; Remold \& Lenski 2004; Weinreich et al. 2005; 2006; Poelwijk et al. 2007; Cooper et al. 2008; Almahmoud et al. 2009; Le Gac \& Doebeli 2010). Beneficial mutations in global regulator-encoding genes likely reorganize the architecture of the cellular regulatory networks, potentially contributing to the influence of epistasis (Khan et al. 2011; Tenaillon et 
al. 2012), and to complex phenotypic changes in environmental conditions distinct from the evolution environment (i.e., correlated responses).

In the present study, we investigated the effect of beneficial mutations on correlated responses to evolution. Replicate populations evolving in identical environments are often characterized by having high phenotypic parallelism. The extent to which this parallelism applies to correlated phenotypic responses is, however, less well studied. At one extreme, replicate populations may display parallel correlated responses in many alternative environments. At the other, they may display varied phenotypes under alternative conditions and phenotypic parallelism only in the evolution environment. These phenotypic outcomes have important ecological implications since they dictate how ecologically similar populations will cope with environmental challenges such as the colonization of new habitats/hosts or the modification of ecosystems at both the biotic and abiotic levels. Only few studies using model bacteria have investigated the correlated phenotypic responses to evolution, including some that revealed parallel losses of catabolic abilities and growth phenotypes on alternative resources (Cooper \& Lenski 2000; Barrett et al. 2005; Fong et al. 2005). However, in other studies using the same bacterial species, populations have been found to be more variable when assayed for growth on alternative resources than on the resource on which they first evolved (Travisano et al. 1995; Travisano \& Lenski 1996; MacLean \& Bell 2003; Ostrowski et al. 2005; 2008). The genetic changes associated with adaptation to a given evolution environment have only rarely been investigated for their effects on correlated responses in alternative environments. Intuitively, one may expect the correlated responses to be more similar for clones sharing mutated genes, but the relationship between genetic parallelism and phenotypic performance in alternative environments may be complex. For example, different mutations affecting a given gene may have similar phenotypic effect in the evolution environment but not necessarily in another one, especially in the case of global regulator-encoding genes. 
Conversely, even if replicate populations adapt through mutations in different genes, the phenotypic consequences of these mutations could be similar not only in the evolution environment, but also under alternative environmental conditions.

Here, we propagated four replicate populations of Escherichia coli for 1000 generations in each of four different environments. We sequenced the genome of one evolved clone isolated from each of the 16 populations, allowing us to identify mutations associated with evolution in each environment. We assayed the phenotype of isolated clones in their evolution environment as well as in the three alternative ones to provide a measure of the direct and correlated phenotypic responses to evolution in all four environments. Using this experimental design, we investigated first, the level of genetic parallelism associated with evolution in several environments and second, the respective effect of genetic parallelism and evolutionary history on the correlated responses to evolution. In particular, we tested whether phenotypes in alternative environments (correlated responses to evolution) were more similar for clones sharing mutations in identical genes or genetic loci (genetic parallelism) irrespective of the environment in which they evolved (evolutionary history) or for clones that evolved in the same environment irrespective of genetic parallelism.

\section{Material and methods}

\section{Experimental evolution}

E. coli B REL606 (Lederberg 1966; Jeong et al. 2009) was used as the ancestor to found four replicate populations that were propagated by daily serial transfer in each of four different environments for 1000 generations at $37^{\circ} \mathrm{C}$. Population samples were collected at 100generation intervals and frozen at $-80{ }^{\circ} \mathrm{C}$ as glycerol suspensions in the same conditions as the ancestor. All four environments are based on Davis minimal (DM) medium (Lenski et al. 1991). The first environment, named Ace, comprised $15 \mathrm{~mL}$ DM supplemented with $2 \mathrm{~g} / \mathrm{L}$ 
113 sodium acetate trihydrate in 50-mL flasks shaken at $200 \mathrm{rpm}$. The second, named Gly,

114 comprised $15 \mathrm{~mL}$ DM supplemented with $1 \mathrm{~g} / \mathrm{L}$ glycerol in static Petri dishes. The third,

115 named Glc, comprised $15 \mathrm{~mL}$ DM supplemented with $1 \mathrm{~g} / \mathrm{L}$ D-gluconate in test tubes shaken

116 at $200 \mathrm{rpm}$. The fourth, named Glu, comprised $600 \mu \mathrm{L}$ DM supplemented with $1 \mathrm{~g} / \mathrm{L}$ D-

117 glucose in $1 \mathrm{~mL}$ x 96-well plates shaken at $200 \mathrm{rpm}$. These four environments were chosen in

118 order to apply a combination of parameters impacting bacterial growth and adaptation due to

119 contrasting carbon sources, and homogenization and oxygenation levels. This choice was

120 made $a$ priori to allow a wide range of mutational targets, including genes associated with

121 metabolic pathways, such as the catabolism of a specific carbon source, but also regulatory

122 genes that influence a combination of growth aspects such as carbon source consumption and

123 better oxygen use. This strategy should also favor the selection of mutations with large

124 correlated phenotypic effects in alternative environments. Every day ( $24+/-2$ hours),

125 populations were diluted 300 -fold into the same fresh medium, allowing $~ 8.2\left[\log _{2}(300)\right]$

126 generations per day. Populations therefore experience every day a lag phase with no

127 detectable growth, followed by an exponential phase characterized by the optimal

128 consumption of the available carbon source, and finally a stationary phase when the carbon

129 source has been exhausted (except in the Ace environment, see below), before being diluted

130 again in fresh medium. The bottleneck at each daily transfer was never fewer than $\sim 4 \times 10^{6}$

131 cells in any environment. After 1000 generations, each of the 16 populations was streaked on

132 LB agar plates that were incubated overnight at $37^{\circ} \mathrm{C}$. A single colony was randomly chosen

133 from each plate and frozen at $-80{ }^{\circ} \mathrm{C}$ as a glycerol suspension.

135 Growth profile assays

136 Growth profiles were measured first for population samples collected every 200 generations,

137 in triplicate and in their respective evolution environments, and second for the ancestor and 
138 individual evolved clones with five-fold replication and in the four different environments.

139 For each experiment, a physiological pre-acclimation was performed in the assay

140 environment, consisting of an overnight culture of bacterial cells followed by a 300-fold

141 dilution and a 24-h incubation. This pre-acclimation step differed slightly for the growth

142 profiles in the Ace environment, in which populations that evolved in the three other

143 environments, as well as the ancestor, grew slowly and were still in exponential phase after 24

144 hours of incubation. In this particular case, the initial overnight cultures were performed in

145 Glu. For all experiments and after a 300-fold dilution, pre-acclimated cultures were incubated

146 in the relevant assay environment.

147 Growth profiles were determined by measuring the optical density for each culture at $600 \mathrm{~nm}$

$148\left(\mathrm{OD}_{600}\right)$ at regular intervals during $24 \mathrm{~h}$ of incubation. We used the resulting growth curves to

149 calculate the maximum growth rate $\left(\mu_{\max }\right)$ of each evolved culture (populations and individual

150 clones) relative to the ancestor. For the Gly, Glc and Glu environments, maximum growth

151 rates were measured between 0.2 and 0.8 of the maximal ancestral $\mathrm{OD}_{600}$. In the Ace

152 environment, the ancestor was still in exponential phase after $24 \mathrm{~h}$ of culture, and growth rates

153 were measured between 0.5 and 1 times the $\mathrm{OD}_{600}$ reached by the ancestor following $24 \mathrm{~h}$ of

154 growth.

155

$156 \quad$ Fitness assays

157 Fitness assays were performed for the populations sampled at 300, 600 and 1000 generations

158 in their respective evolution environments, and for the individual evolved clones in all four

159 environments. All assays were replicated five-fold. Competitions were performed as

160 previously described (Lenski et al. 1991). Briefly, all competitors including the REL606

161 ancestor and a marked phenotypic variant called REL607 were pre-acclimated in the assay

162 environment. The ancestor REL606 and all derived evolved clones are unable to use 
arabinose as a carbon source (Ara'), while REL607 is a REL606 spontaneous revertant that recovered this catabolic ability $\left(\mathrm{Ara}^{+}\right)$. After pre-acclimation, each evolved sample (population and individual clones) and the REL606 ancestral strain as a control were mixed separately with REL607 at a 1:1 ratio. Mixtures were then diluted 300-fold in fresh medium and incubated for $24 \mathrm{~h}$ at $37^{\circ} \mathrm{C}$ in the assay environment. At days 0 (when the two competitors are mixed) and 1 (after $24 \mathrm{~h}$ of incubation) of each competition experiment, cells were diluted and plated on indicator tetrazolium arabinose (TA) plates, on which $\mathrm{Ara}^{+}$or $\mathrm{Ara}^{-}$ colonies appear pink or red, respectively (Lenski et al. 1991). Plates were incubated 24 h at 37 ${ }^{\circ} \mathrm{C}$ and each of the competitors was scored. Using the initial and final cell counts we calculated the realized (net) population growth of each competitor, according to the following formula: $G_{i}=\ln \left(C_{t 1} * 300 / C_{t 0}\right)$, where $C_{t 0}$ and $C_{t 1}$ are the number of colonies at the beginning and after 24 hours of competition, respectively, and 300 the 300 -fold higher dilution factor required for $\mathrm{C}_{\mathrm{t} 1}$ compared to $\mathrm{C}_{\mathrm{t} 0}$. The fitness of one competitor relative to the other was then calculated as the ratio of their net growth rates during the competition experiment according to the formula: Fitness $=\mathrm{G}_{\mathrm{Ara}}-\mathrm{G}_{\mathrm{Ara}}+$, where $\mathrm{G}_{\mathrm{Ara}}-$ and $\mathrm{G}_{\mathrm{Ara}}+$ are the realized population growth of the Ara ${ }^{-}$ancestor and evolved clones and of the Ara ${ }^{+}$REL607 clone, respectively (Lenski et al. 1991).

\section{Genome sequencing}

The genome of each of the 16 clones isolated after 1000 generations of evolution was resequenced on the Illumina HiSeq2000 platform (GATC Biotech, Germany) using one lane of single-end 35-bp reads. Barcodes were used for each genome so that reads were clonespecific. Candidate point mutations were identified in comparison to the ancestral genome of REL606 (Jeong et al. 2009) using the SNiPer pipeline (Wielgoss et al. 2011). The existence of a mutational event was inferred when it was identified in more than $75 \%$ of the reads 
covering a given site and in more than 20 reads. We identified a total of 54 mutations of which 25 were randomly chosen and confirmed by PCR and sequencing of the obtained products. Short read single-end re-sequencing reliably detects SNPs but may miss large indels and mutational events associated with mobile genetic elements (recombination and transposition). To minimize this potential bias, we checked the eight genes that had a mutation in more than one clone, i.e. mutated in parallel, for gene size polymorphism by PCR amplification. Using this approach, we identified two additional mutations in the $g l p R$ gene: one IS1 insertion 30 bp upstream of its start codon and one 125-bp deletion in its coding region. Re-sequencing the genome of one single evolved clone sampled from each population allowed us to directly link phenotypic changes in different environments to specific combinations of mutations. This approach is not appropriate for analyzing genomic evolution at the level of the entire populations which is beyond the scope of our study and would imply re-sequencing genomes from either multiple evolved clones or mixtures of populations (Barrick \& Lenski 2009; Herron \& Doebeli 2013).

\section{Statistical analyses}

As the same global regulator-encoding genes were mutated in more than one environment, we used a generalized linear model (GLM) to test if the correlated responses were more similar for clones that shared mutated genes and/or that evolved in the same environments. To build the model, we measured the variability of the phenotypes (average $\mu_{\max }$ and fitness values) between clones in their alternative environments. For pairs of clones that evolved in the same environment we considered the three alternative environments, while for those that evolved in two different environments we considered the two shared alternative ones. The variability of the correlated responses was calculated as the mean squares among clones (MSS), as for an ANOVA, but we did not compare the MSS to the mean squares error (MSE) using F statistics. 
Instead, MSS were ranked within each alternative environment and used as an ordinal

214 response variable in the GLM. By doing this, we could consider as the units of interest the

215 pairs of clones instead of the clones themselves, allowing for the choice of two explanatory

216 variables. The first distinguished the pairs of clones sharing or not a mutated gene (nominal

217 explanatory variable) and the second the pairs of clones that evolved or not in the same

218 environment (nominal explanatory variable). Details of the input data used for the GLM are

219 given in a Dryad file (doi:10.5061/dryad.n2582). Likelihood ratio $\chi^{2}$ values were used to test

220 for significance of ordinal logistic regressions as implemented in JMP version 3.1.5 (SAS

221 Institute).

Results

Phenotypic changes in the evolution environments

Four replicate populations of $E$. coli B were propagated for 1000 generations in each of four

evolution environments that differed in carbon source, oxygenation and degree of spatial

structure. We quantified the phenotypic evolution of each population in its respective

evolution environment by measuring its maximum growth rate relative to the ancestor at 200generation intervals ( $\mu_{\max }$; Fig. 1a), and its fitness relative to the ancestor at 300, 600 and 1000 generations (Fig. 1b). Compared to their common ancestor all populations increased in

231 fitness and all except those evolved in Glu increased their $\mu_{\max }$ after 1000 generations,

232 indicating that they adapted to their environments (Table S1, Supporting information, Fig. 1).

233 After 1000 generations of evolution, we isolated one evolved clone from each replicate

234 population and measured $\mu_{\max }$ and fitness in its evolution environment (Table S2, Supporting

235 information, Fig. 2). Phenotypic evolution of the clones and their source populations was

236 correlated $\left(\mu_{\max }:\right.$ Spearman Rho $=0.93, p<0.0001$; fitness: Spearman Rho $=0.60, p=$

237 0.0144). However, the clones isolated from the Glu environment tended to display a higher 
$\mu_{\max }$ than their entire source populations, indicating that they probably belong to specific sub-

239 lineages that may not be representative of the entire populations. This has however no impact

240 on our results since our main goal here is to relate phenotypes to specific combinations of

241 mutations, a task that could not be achieved by focusing on entire populations. Therefore, all

242 the following analyses focus on comparisons between phenotypes and genomes of clones and 243 not populations.

244 Clones sampled from the four environments had quantitative differences in their divergence 245 from the ancestor (Kruskal-Wallis one-way analysis of variance: $\mu_{\max }, \chi^{2}=12.71, p=0.0053$; 246 fitness, $\left.\chi^{2}=11.59, p=0.0089\right)$, indicating different magnitudes of phenotypic evolution in the 247 four environments (Table S2, Supporting information, Fig. 2).

\section{Genome sequencing}

Re-sequencing the genomes of the 16 evolved clones revealed a total of 54 mutations

compared to the ancestor, with one to six mutations for each clone. These mutations affected a occurred within genes, including 49 non-synonymous and only 2 synonymous changes. Only three mutations affected intergenic regions, including two SNPs and the 1-bp insertion. Two additional indels were subsequently identified (see Material and methods), one IS 1 insertion and one 125-bp deletion, both affecting the $g l p R$ gene (Table 1).

Genetic parallelism within and between evolution environments

259 Defining genetic parallelism as a mutational change occurring in identical genes, operons or 260 functional units (Tenaillon et al. 2012) in at least two clones, $60 \%(34 / 56)$ of the identified 261 mutations occurred in parallel (Fig. 3, Table 1). The proportion of parallel to total mutations 262 was 7/12, 13/19, 9/16, and 6/9 in the Ace, Gly, Glc, and Glu environments, respectively. This 
parallelism involved eight genes or operons (Fig. 3, Table 1), including four specific to a

264 single environment ( $m r e B C$ in Ace, $g l p R$ and $g l p K$ in Gly, and $l l d R$ in Glc), and four across multiple environments $(\arg R, \operatorname{spoT}, \mathrm{rho}$, and $\operatorname{nadR})$. In all cases genes that changed in parallel

within a single environment reflected a greater degree of environmental clustering than expected if the mutations were distributed randomly over the evolved clones (Fisher's exact test, for all genes mutated in parallel $p \leq 0.05)$. These data are consistent with the changes in 269 these genes conferring environment-specific adaptations.

270 The four evolved clones sampled from the Ace environment had a total of 12 mutations 271 affecting nine different genes that can be grouped into eight loci. Three of these loci were 272 targeted by mutations in more than one clone: the $m r e B C$ genetic locus, $m r e B$ and $m r e C$ being 273 part of the same operon (Wachi et al. 2006), had mutations in two clones, while $r h o$ and $\operatorname{argR}$ 274 were mutated in three and two clones, respectively (Fig. 3, Table 1). The four clones sampled 275 from the Gly environment had a total of 19 mutations affecting ten different genes. Seven 276 mutations occurred in $g l p R$ and $g l p K$, which were mutated in four and three clones, 277 respectively, while six occurred in spoT and rho, which were mutated in four and two clones, 278 respectively (Fig. 3, Table 1). The four evolved clones sampled from the Glc environment had 279 a total of 16 mutations affecting ten different genes, among which three ( $\arg R, l l d R$ and spoT) 280 had mutations in three clones (Fig. 3, Table 1). Finally, the four evolved clones sampled from 281 the Glu environment had 9 mutations affecting a total of 5 different genes including spoT and $282 n a d R$ that were mutated in four and two clones, respectively (Fig. 3, Table 1). Despite the 283 high overall level of genetic parallelism, the number of parallel changes found in individual 284 clones was quite variable (Fig. 3, Table 1), ranging from one (Ace_4, Glc_2 and Glu_2) to 285 four (Gly_3, Glc_4, Glu_1 and Glu_4). 
287 Four genes were mutated repeatedly in different environments—argR (Ace and Glc

288 environments), spoT (Gly, Glc and Glu environments), rho (Ace and Gly environments), and $289 \operatorname{nadR}$ (Gly and Glu). Therefore, evolved clones from all pairs of environments, except

290 Ace/Glu, shared mutated genes, but the combinations of shared mutated genes were

291 environment-specific (Fig. 3, Table 1). The highest level of genetic parallelism was detected

292 for spoT, which was modified in 3/4 environments and 11/16 clones, further emphasizing

293 previous observations of repeated spoT mutations (see below; Cooper et al. 2003; Cooper

294 2007; Ostrowski et al. 2008; Woods et al. 2011).

295

Functions of mutated genes and distribution of mutations within repeatedly modified genes

297 We investigated the function of the mutated genes by considering their Gene Ontology

298 categories (Riley et al. 2006). Seven (rpoA, rho, $\operatorname{argR}, l l d R, g l p R, f l h D$, and $n a d R$ ) of the 25

299 mutated genes are categorized as transcription regulators, which is more than expected by

300 chance alone (Fisher one tail exact test $p=0.01$ ). Although not classified in the GO

301 transcription category, other mutated genes are also involved in transcription regulation: fabR

302 encodes a transcription regulator, and $\mathrm{relA}$ and spoT are involved in the metabolism of

303 ppGpp, which reprograms the entire transcription machinery in bacterial cells (Srivatsan \&

304 Wang 2008). Therefore, 10 of the 25 genetic loci that were modified during evolution are

305 involved in transcription regulation. Strikingly, six of them (rho, argR, lldR, glpR, nadR, and

306 spoT) are among the eight that were mutated in parallel, emphasizing the importance of

307 changes in transcription regulation for adaptation.

308 Two sub-groups can be distinguished among these six genes. The first comprises $g l p R$ and

$309 l l d R$, which are local transcription regulators (Zeng et al. 1996; Aguilera et al. 2008) affecting

310 the expression of a small number of genes involved in the consumption of a single carbon

311 source (glycerol or lactate, respectively). Parallel changes occurred in these two genes only 
312 within specific environments (Gly or Glc, respectively). The second sub-group comprises

$313 \operatorname{argR}$, which regulates a large set of target genes (Caldara et al. 2006), spoT and rho, which

314 are involved in global gene regulation (Srivatsan \& Wang 2008; Epshtein et al. 2010), and

$315 n a d R$, which regulates global cell physiology by integrating multiple environmental signals to

316 control the synthesis of NAD, a central co-factor in many bacterial metabolic pathways

317 (Grose et al. 2005). Interestingly, it is precisely this second group of transcription regulator-

318 encoding genes that had parallel changes between, as well as within, environments.

319 The genetic parallelism observed at the gene level was associated with mutations affecting

320 different codons largely distributed within these genes, with however two notable exceptions

321 (Table 1). First, specific regions of the same genes were affected by independent mutations,

322 including the 5' end of $\operatorname{argR}$ and $g l p K$. Second, nearby codons were affected by independent 323 non-synonymous mutations in rho (codons 322 and 324), and spoT (codons 207 and 209). In

324 addition, the exact same codons of rho (codons 322 and 324) and spoT (codon 393) were 325 repeatedly affected by independent mutations, leading to either the same or different amino326 acid changes in the corresponding proteins (Table 1). For both exceptions, similar mutations 327 occurred in more than one environment.

Correlated phenotypic responses to evolution in the alternative environments

330 Next, we investigated how adaptation to a given evolution environment affected $\mu_{\max }$ and

331 fitness in alternative conditions. The correlated response to selection of each of the 16 evolved

332 clones was compared to the common ancestor in each of the three environments in which it 333 did not evolve (hereafter called alternative environments). As an example, the four evolved 334 clones sampled from the populations that were propagated in Ace were assayed in the 335 alternative environments Gly, Glc and Glu. 
336 Growth profiles and competition assays indicated that adaptation to a given environment was

337 often associated with improvements in the alternative environments (Table S2, Supporting

338 information, Fig. 4). Phenotypic evolution in a given environment is constrained by selective

339 pressures that need not to apply in alternative environments. Therefore, we expected the

340 correlated phenotypic responses in the alternative environments to be more variable than the

341 direct response in the evolution environment. Surprisingly, this was not the case (Wilcoxon

342 rank-sum test on the coefficient of variation; $\mu_{\max }: \chi^{2}=1.9, p=0.17$; fitness: $\chi^{2}=0.2, p=$

343 0.69; Table S3, Supporting information).

344 Next, we tested whether genetic parallelism (i.e., whether clones shared or not mutated genes)

345 and evolutionary history (i.e., whether clones evolved or not in the same evolution

346 environment) affected the correlated responses. Correlated responses to evolution may be

347 expected to be more similar for clones with mutations in identical genes, even if they evolved

348 in different environments, as long as the phenotypic effects of individual mutations affecting a

349 given gene are similar and epistatic interactions between mutations in the different genes do

350 not entirely mask the effect of individual mutations. Similarly, correlated responses to

351 evolution may be expected to be more similar for clones that evolved in identical

352 environments, even if they had mutations in different genes, if the evolved mutational

353 pathways resulted in phenotypic parallelism not only in the evolution environment but also

354 under a wider range of environments. We measured the variability of the correlated responses

355 in each alternative environment for all pairs of clones and used a GLM to distinguish the

356 contribution of genetic parallelism and evolutionary history to the correlated responses to

357 evolution. For pairs of clones that evolved in the same evolution environment we considered

358 the three alternative environments, while for pairs of clones that evolved in two different

359 evolution environments we considered the two shared alternative environments. We tested

360 whether sharing or not mutated genes and evolving or not in identical evolution environments 
361 influenced the phenotypic variability between clone pairs (Table 2). The interaction between

362 the two factors was significant for both $\mu_{\max }$ and fitness phenotypes (Table 2), indicating that

363 the impact of genetic parallelism on phenotypic variability was different whether the clones

364 evolved in identical or different evolution environments. The variability of the correlated

365 responses was lower for pairs of clones that evolved in the same environment (Fig. 5).

366 Moreover, clones with different evolutionary history had more similar correlated responses

367 when they shared mutated genes (Fig. 5a, b). However, for clones that evolved in identical

368 evolution environments, the variability of correlated responses was unrelated to the level of

369 genetic parallelism (Fig. 5c, d).

371 Discussion

372 We propagated four replicate populations of E. coli B in each of four different environments

373 for 1000 generations. During this time all populations adapted to their evolution environment.

374 Adaptation involved a high level of genetic parallelism, and $60 \%$ of the identified mutations

375 affected eight genes that were modified in parallel in at least two populations. Four of these 376 genes, $\arg R$, spoT, rho, and $n a d R$, were modified repeatedly across environments, although

377 no single gene was mutated in all environments. This result suggests distinct, but overlapping,

378 selective pressures in the different environments. Moreover, the combination of mutated

379 genes was different in the evolved clones sampled from the various environments. Finally, we

380 found that evolution in each environment was associated with improved correlated phenotypic

381 responses in alternative environments and that evolved clones from different evolution

382 environments revealed a higher level of parallel correlated responses when displaying genetic 383 parallelism. 
Evolution was associated with parallel genetic changes in genes specifically involved in the

catabolism of the available carbon source $(g l p R$ and $g l p K)$ in only one of the four environments (Gly). Adaptive evolution may therefore involve improvements of substrate specific catabolic pathways, but this not a general trend. Consistent with this, other evolution experiments have found mutations in genes specifically involved in the catabolic pathways of the available carbon sources in populations propagated in the presence of glycerol (Herring et al. 2006) and L-1,2-propanediol (Lee \& Palsson 2010), but not in the presence of lactate (Conrad et al. 2009) or glucose (Barrick et al. 2009). We found that genes involved in global transcription regulation were targets of mutations in all four evolution environments, as observed in most evolution experiments (Cooper et al. 2003; Crozat et al. 2005; Herring et al. 2006; Bantinaki et al. 2007; Conrad et al. 2009; Le Gac \& Doebeli 2010; Maharjan et al. 2010; Wang et al. 2010; Yu et al. 2010; Conrad et al. 2011; Tenaillon et al. 2012), reflecting the importance of restructuring regulatory networks during evolution (Philippe et al. 2007; Hindré et al. 2012). Even more strikingly, all genes that were mutated in more than one environment encoded global regulators of gene transcription. Six of the eight genetic loci that were repeatedly changed have also been modified by mutations in other evolution experiments, including glpK, glpR, spoT, nadR, mreBC and rho (Cooper et al. 2003; Raghunathan \& Palsson 2003; Herring et al. 2006; Woods et al. 2006; Ostrowski et al. 2008; Woods et al. 2011; Tenaillon et al. 2012; Herron and Doebeli 2013).

Genetic parallelism is commonly observed among replicate independent populations that are propagated in similar environments. The nature of the mutations affecting target genes is diverse, from identical nucleotide changes being repeatedly substituted in independent viral populations (Wichman et al. 1999) to different types of mutations distributed at various positions within a given gene in replicate bacterial populations (Woods et al. 2006; Bantinaki 
et al. 2007; Crozat et al. 2010). A recent analysis of 115 replicate populations of $E$. coli B

411 adapted to high temperature revealed few identical mutations among populations, although

412 high genetic parallelism at the gene level was detected (Tenaillon et al. 2012). In our study,

413 genetic parallelism at the gene level was associated with different mutations occurring at

414 different positions within genes. For example, $g l p R$ was affected by two different non-

415 synonymous mutations, a 125-bp deletion in its coding region, and the insertion of an IS 1

416 element in its promoter region. This profile suggests that the mutations inactivated $g l p R$.

417 However, there were two notable exceptions to this pattern. First, different mutations occurred

418 in specific regions of two of the altered genes. All $\operatorname{argR}$ mutations were localized in the 5'

419 gene region encoding the DNA-binding domain of ArgR (Sunnerhagen et al. 1997) and all

420 glpK mutations mapped in a $\sim 80$-bp region at the 5' end of the gene. Mutations in this glpK

421 region have been shown to increase fitness on glycerol-containing medium by reducing the

422 affinity of GlpK for its allosteric inhibitor fructose-1,6-bisphosphate and inhibiting the

423 formation of the GlpK tetramer (Applebee et al. 2011). Based on these observations, we

424 hypothesize that mutations in $\arg R$ and $g l p K$ modulated specific activities of the encoded

425 proteins. Second, we detected an even higher level of specificity for the mutations that

426 occurred in rho and spoT, which were repeatedly modified both within and between

427 environments. Hence, not only were independently substituted mutations localized to nearby

428 codons (322 and 324 in rho, 207 and 209 in spoT), but identical codons were changed in

429 clones evolved in different environments, including codons 322 and 324 for rho in Ace and

430 Gly and codon 393 for spoT in Gly and Glu. In the Gly environment, codon 393 of spoT was

431 changed in all four clones, and in 3 out of 4 in Glu. Cross contamination between replicate

432 populations can be excluded since four different SNPs affected codon 393. Such an extreme

433 level of parallelism, extending to the level of the substituted mutations, has also been reported

434 in bacterial evolution experiments for $g l p K$ (Applebee et al. 2011), rho (Tenaillon et al. 
2012), and genes that are the targets for antibiotic resistance (Martinez et al. 2011). Therefore,

436 depending on the environment, selection may act either at precise identical codons (in Gly and

437 Glu), leading or not to identical amino-acid changes, or at the level of the entire gene (Glc).

438 In our evolution experiment, spoT was the most commonly mutated gene, being modified in

$4393 / 4$ environments and 11/16 clones. It has already been observed as being frequently modified

440 during adaptation to glucose environments. In a commonly-used glucose environment, spoT

441 has been affected by a total of 32 non-synonymous mutations, 28 of which involved a unique

442 amino-acid substitution (Cooper et al. 2003; Cooper 2007; Ostrowski et al. 2008; Woods et

443 al. 2011). In contrast, in two environments in this study, seven out of eight clones had

444 mutations affecting the exact same spoT codon. This suggests that the number of potential

445 beneficial mutations in a given gene may vary from one environment to the other. So far, very

446 little is known about the variability of phenotypic effects associated with different beneficial

447 mutations affecting the same gene. Such studies would undoubtedly increase our

448 comprehension of the relationships between genotypes, phenotypes and fitness.

450 We have shown that populations evolving in identical evolution environments may display

451 parallel phenotypic evolution in alternative environments, as was previously described

452 (Cooper \& Lenski 2000; Barrett et al. 2005; Fong et al. 2005). Moreover, we showed that the

453 correlated phenotypic responses were related to both the evolutionary history of the sampled

454 clones, i.e. whether or not they evolved in identical evolution environments, and the genetic

455 parallelism, i.e. whether or not they shared mutated genes. Our results therefore have two

456 implications. First, individuals that have been subjected to similar historical selective

457 pressures tend to occupy similar ecological niches not only in the environment in which they

458 evolved, but also under a wider range of conditions, even without genetic parallelism. Second,

459 when evolutionary history was different, i.e. for clones that evolved in different evolution 
environments, correlated phenotypic responses were related to the level of genetic parallelism.

461 It therefore suggests that the mutations of the repeatedly modified genes had similar beneficial

462 effects even in different genomic contexts. This finding indicates that although epistatic

463 interactions between mutations are known to considerably alter the effect of individual

464 mutations, they evidently did not entirely mask the individual effects of the mutations under

465 our environmental conditions.

466 In summary, notwithstanding the relatively small number of genes mutated in more than one

467 environment, our results provide a way to experimentally disentangle the relative contribution

468 of evolutionary history and genetic parallelism on the phenotypic performance in naïve

469 environmental conditions. Taken together, our results indicate that populations sharing

470 historical selective pressures could react similarly to the modification of environmental

471 conditions, even if they do not share mutations in specific genes. To a lesser extent, they also

472 indicate that populations with different evolutionary histories may perform more similarly

473 when facing a new environment if they share mutated genes.

474

475 Acknowledgements

476 This research was supported by the French Centre National de la Recherche Scientifique

477 (CNRS), the University Joseph Fourier Grenoble and by a grant from the Agence Nationale

478 de la Recherche (ANR, Program Blanc, Grant ANR-08-BLAN-0283-01 to D. Schneider) and

479 the NSF (DEB0844355 to T. F. Cooper). M. Le Gac thanks the ANR for a post-doctoral 480 fellowship. 


\section{References}

483 Aguilera L, Campos E, Gimenez R, et al. (2008) Dual role of LldR in regulation of the 484 IldPRD operon, involved in L-lactate metabolism in Escherichia coli. Journal of 485 Bacteriology, 190, 2997-3005.

486 Almahmoud I, Kay E, Schneider D, Maurin M (2009) Mutational paths towards increased 487 fluoroquinolone-resistance in Legionella pneumophila. Journal of Antimicrobial $488 \quad$ Chemotherapy, 64, 284-293.

489 Applebee MK, Joyce AR, Conrad TM, Pettigrew DW, Palsson BO (2011) Functional and 490 metabolic effects of adaptive glycerol kinase (GLPK) mutants in Escherichia coli. Journal 491 of Biological Chemistry, 286, 23150-23159.

492 Bantinaki E, Kassen R, Knight CG, et al. (2007) Adaptive divergence in experimental 493 populations of Pseudomonas fluorescens. III. Mutational origins of wrinkly spreader 494 diversity. Genetics, 176, 441-453.

495 Barrett RDH, MacLean RC, Bell G (2005) Experimental evolution of Pseudomonas 496 fluorescens in simple and complex environments. American Naturalist, 166, 470-480.

497 Barrick JE, Lenski RE (2009) Genome-wide mutational diversity in an evolving population of 498 Escherichia coli. Cold Spring Harbor Symposium Quantitative Biology, 74, 119-129.

499 Barrick JE, Yu DS, Yoon SH, et al. (2009) Genome evolution and adaptation in a long-term $500 \quad$ experiment with Escherichia coli. Nature, 461, 1243-1247.

501 Brockhurst MA, Colegrave N, Rozen DE (2011) Next-generation sequencing as a tool to 502 study microbial evolution. Molecular Ecology, 20, 972-980.

503 Bull JJ, Badgett MR, Wichman HA (2000) Big-benefit mutations in a bacteriophage inhibited $504 \quad$ with heat. Molecular Biology and Evolution, 17, 942-950. 
Caldara M, Charlier D, Cunin R (2006) The arginine regulon of Escherichia coli: whole-

506 system transcriptome analysis discovers new genes and provides an integrated view of 507 arginine regulation. Microbiology, 152, 3343-3354.

508 Conrad TM, Joyce AR, Applebee MK, et al. (2009) Whole-genome resequencing of

509 Escherichia coli K-12 MG1655 undergoing short-term laboratory evolution in lactate

510 minimal media reveals flexible selection of adaptive mutations. Genome Biology, 10, R118.

511 Conrad TM, Lewis NE, Palsson BO (2011) Microbial laboratory evolution in the era of 512 genome-scale science. Molecular and Systems Biology, 7, 509.

513 Cooper TF, Remold SK, Lenski RE, Schneider D (2008) Expression profiles reveal parallel 514 evolution of epistatic interactions involving the CRP regulon in Escherichia coli. PLoS $515 \quad$ Genetics, 4(2), e35.

516 Cooper TF, Rozen DE, Lenski RE (2003) Parallel changes in gene expression after 20,000 517 generations of evolution in Escherichia coli. Proceedings of the National Academy of $518 \quad$ Sciences, USA, 100, 1072-1077.

519 Cooper TF (2007) Recombination speeds adaptation by reducing competition between 520 beneficial mutations in populations of Escherichia coli. PLoS Biology, 5(9), e225.

521 Cooper VS, Lenski RE (2000) The population genetics of ecological specialization in 522 evolving Escherichia coli populations. Nature, 407, 736-739.

523 Crozat E, Philippe N, Lenski RE, Geiselmann J, Schneider D (2005) Long-term experimental 524 evolution in Escherichia coli. XII. DNA topology as a key target of selection. Genetics, $525 \quad \mathbf{1 6 9}, 523-532$.

526 Crozat E, Winkworth C, Gaffé J, et al. (2010) Parallel genetic and phenotypic evolution of 527 DNA superhelicity in experimental populations of Escherichia coli. Molecular Biology and $528 \quad$ Evolution, 27, 2113-2128. 
Dettman JR, Rodrigue N, Melnyk AH, Wong A, Bailey SF, Kassen R (2012) Evolutionary

530 insight from whole-genome sequencing of experimentally evolved microbes. Molecular Ecology, 21, 2058-2077.

532 Epshtein V, Dutta D, Wade J, Nudler E (2010) An allosteric mechanism of Rho-dependent 533 transcription termination. Nature, 463, 245-249.

534 Fong SS, Joyce AR, Palsson BO (2005) Parallel adaptive evolution cultures of Escherichia 535 coli lead to convergent growth phenotypes with different gene expression states. Genome $536 \quad$ Research, 15, 1365-1372.

537 Grose JH, Bergthorsson U, Roth JR (2005) Regulation of NAD biosynthesis by the 538 trifunctional NadR protein of Salmonella enterica. Journal of Bacteriology, 187, 27745392782.

540 Herring CD, Raghunathan A, Honisch C, et al. (2006) Comparative genome sequencing of 541 Escherichia coli allows observation of bacterial evolution on a laboratory timescale. Nature 542 Genetics, 38, 1406-1412.

543 Herron MD, Doebeli M (2013) Parallel evolutionary dynamics of adaptive diversification in $544 \quad$ Escherichia coli. PLoS Biology, 11, e1001490.

545 Hindré T, Knibbe C, Beslon G, Schneider D (2012) New insights into bacterial adaptation 546 through in vivo and in silico experimental evolution. Nature Reviews Microbiology, 10, $547 \quad 352-365$.

548 Jeong H, Barbe V, Lee CH, et al. (2009) Genome sequences of Escherichia coli B strains 549 REL606 and BL21(DE3). Journal of Molecular Biology, 394, 644-652.

550 Khan AI, Dinh DM, Schneider D, Lenski RE, Cooper TF (2011) Negative epistasis between 551 beneficial mutations in an evolving bacterial population. Science, 332, 1193-1196.

552 Lederberg S (1966) Genetics of host-controlled restriction and modification of 553 deoxyribonucleic acid in Escherichia coli. Journal of Bacteriology, 91, 1029-1036. 
554 Lee DH, Palsson BO (2010) Adaptive evolution of Escherichia coli K-12 MG1655 during

555 growth on a nonnative carbon source, L-1,2-propanediol. Applied and Environmental

$556 \quad$ Microbiology, 76, 4158-4168.

557 Le Gac M, Doebeli M (2010) Epistasis and frequency dependence influence the fitness of an 558 adaptive mutation in a diversifying lineage. Molecular Ecology, 19, 2430-2438.

559 Lenski RE, Rose MR, Simpson SC, Tadler SC (1991) Long-term experimental evolution in

560 Escherichia coli. I. Adaptation and divergence during 2,000 generations. American $561 \quad$ Naturalist, 138, 1315-1341.

562 MacLean RC, Bell G (2003) Divergent evolution during an experimental adaptive radiation.

563 Proceedings of the Royal Society of London series B-Biological Sciences, 270, 1645-1650.

564 Maharjan R, Zhou Z, Ren Y, et al. (2010) Genomic identification of a novel mutation in $h f q$ 565 that provides multiple benefits in evolving glucose-limited populations of Escherichia coli. $566 \quad$ Journal of Bacteriology, 192, 4517-4521.

567 Martinez JL, Baquero F, Andersson DI (2011) Beyond serial passages: new methods for 568 predicting the emergence of resistance to novel antibiotics. Current Opinion in 569 Pharmacology, 11, 439-445.

570 Ostrowski EA, Rozen DE, Lenski RE (2005) Pleiotropic effects of beneficial mutations in $571 \quad$ Escherichia coli. Evolution, 59, 2343-2352.

572 Ostrowski EA, Woods RJ, Lenski RE (2008) The genetic basis of parallel and divergent 573 phenotypic responses in evolving populations of Escherichia coli. Proceedings of the Royal $574 \quad$ Society of London series B-Biological Sciences, 275, 277-284.

575 Philippe N, Crozat E, Lenski RE, Schneider D (2007) Evolution of global regulatory networks 576 during a long-term experiment with Escherichia coli. BioEssays, 29, 846-860.

577 Poelwijk FJ, Kiviet DJ, Weinreich DM, Tans SJ (2007) Empirical fitness landscapes reveal 578 accessible evolutionary paths. Nature, 445, 383-386. 
Raghunathan A, Palsson BO (2003) Scalable method to determine mutations that occur during $580 \quad$ adaptive evolution of Escherichia coli. Biotechnology Letters, 25, 435-441.

581 Remold SK, Lenski RE (2004) Pervasive joint influence of epistasis and plasticity on 582 mutational effects in Escherichia coli. Nature Genetics, 36, 423-426.

583 Riley M, Abe T, Arnaud MB, et al. (2006) Escherichia coli K-12: a cooperatively developed 584 annotation snapshot—2005. Nucleic Acids Research, 34, 1-9.

585 Srivatsan A, Wang JD (2008) Control of bacterial transcription, translation and replication by 586 (p)ppGpp. Current Opinion in Microbiology, 11, 100-105.

587 Sunnerhagen M, Nilges M, Otting G, Carey J (1997) Solution structure of the DNA-binding 588 domain and model for the complex of multifunctional hexameric arginine repressor with 589 DNA. Nature Structural Biology, 4, 819-826.

590 Tenaillon O, Rodriguez-Verdugo A, Gaut RL, et al. (2012) The molecular diversity of $591 \quad$ adaptive convergence. Science, 335, 457-461.

592 Travisano M, Lenski RE (1996) Long-term experimental evolution in Escherichia coli.IV. 593 Targets of selection and the specificity of adaptation. Genetics, 143, 15-26.

594 Travisano M, Vasi F, Lenski RE (1995) Long-term experimental evolution in Escherichia 595 coli. III. Variation among replicate populations in correlated responses to novel 596 environments. Evolution, 49, 189-200.

597 Wachi M, Osaka K, Kohama T, et al. (2006) Transcriptional analysis of the Escherichia coli $598 \quad m r e B C D$ genes responsible for morphogenesis and chromosome segregation. Bioscience, 599 Biotechnology, and Biochemistry, 70, 2712-2719.

600 Wang L, Spira B, Zhou Z, et al. (2010) Divergence involving global regulatory gene 601 mutations in an Escherichia coli population evolving under phosphate limitation. Genome 602 Biology and Evolution, 2, 478-487. 
603 Weinreich DM, Delaney NF, Depristo MA, Hartl DL (2006) Darwinian evolution can follow

604 only very few mutational paths to fitter proteins. Science, $\mathbf{3 1 2}, 111-114$.

605 Weinreich DM, Watson RA, Chao L (2005) Perspective: Sign epistasis and genetic constraint 606 on evolutionary trajectories. Evolution, 59, 1165-1174.

607 Wichman HA, Badgett MR, Scott LA, Boulianne CM, Bull JJ (1999) Different trajectories of 608 parallel evolution during viral adaptation. Science, 285, 422-424.

609 Wielgoss S, Barrick JE, Tenaillon O, et al. (2011) Mutation rate inferred from synonymous 610 substitutions in a long-term evolution experiment with Escherichia coli. G3: Genes, 611 Genomes, Genetics, 1, 183-186.

612 Woods R, Schneider D, Winkworth CL, Riley MA, Lenski RE (2006) Tests of parallel 613 molecular evolution in a long-term experiment with Escherichia coli. Proceedings of the $614 \quad$ National Academy of Sciences, USA, 103, 9107-9112.

615 Woods R, Barrick JE, Cooper TF, et al. (2011) Second-order selection for evolvability in a 616 large Escherichia coli population. Science, 331, 1433-1436.

617 Yu YT, Yuan X, Velicer GJ (2010) Adaptive evolution of an sRNA that controls Myxococcus 618 development. Science, 328, 993.

619 Zeng G, Ye S, Larson TJ (1996) Repressor for the sn-glycerol 3-phosphate regulon of 620 Escherichia coli K-12: primary structure and identification of the DNA-binding domain. $621 \quad$ Journal of Bacteriology, 178, 7080-7089.

\section{Data Accessibility:}

624 Genome sequencing data: ENA Sequence Read Archive (ERP001471)

625 Fitness, growth, and GLM data: DRYAD data identifier:doi:10.5061/dryad.n2582

626 Supporting information

627 Additional supporting information may be found in the online version of this article. 
628 Table S1 Phenotypic traits ( $\mu_{\max }$ and fitness) of the 16 populations during evolutionary time

629 in their respective evolution environments.

630 Table S2 Phenotypic traits ( $\mu_{\max }$ and fitness) of the evolved clones sampled from each of the

63116 populations in the four environments.

632 Table S3 Among-clone coefficient of variation in the four environments for the two

633 phenotypic traits $\mu_{\max }$ and fitness.

634

635 Figure Legends

636 Fig. 1 Phenotypic trajectories of 16 evolving populations during 1000 generations in their

637 respective evolution environments. Average $\mu_{\max }$ (a) and fitness (b) (+/- SEM) are indicated

638 at, respectively, 200-generation intervals, and 300, 600 and 1000 generations for the replicate

639 populations that evolved in the environments Ace (- $\left.\bigcirc^{--}\right)$, Gly $(-\triangle-$ ), Glc $(\cdots \square \cdots \cdots)$, and Glu

640<smiles>[CH]CCCCCC</smiles>

641

642 Fig. 2 Phenotypic traits of the evolved clones sampled from the replicate populations that

643 were propagated in the environments Ace $(\bigcirc)$, Gly $(\Delta)$, Glc $(\square)$, and Glu $(\diamond)$. The fitness

644 and growth rate $\left(\mu_{\max }\right)$ values were measured for one evolved clone, sampled from each

645 population after 1000 generations, in its respective evolution environment. Values were log-

646 transformed and given in the two-dimensional phenotypic space. Each experiment was

647 performed with five-fold replication and values are given +/-SEM. The phenotypic values for

648 the ancestor are $(0 ; 0)$.

649

650 Fig. 3 Venn diagrams showing the mutated genes identified in the 16 evolved clones. Each of

651 the 16 ellipses represents one evolved clone sampled from each of the 16 populations. Red,

652 blue, green, and purple ellipses indicate the environments Ace, Gly, Glc, and Glu, 
653

respectively. Overlaps indicate mutated genes shared between clones. Names of genes are

654 written in different colours whether they are affected by mutations in at least two different environments (white), or in only one environment either in a single (black) or in different clones in that environment (colour corresponding to the specific environment).

657

658 quartiles (_.....) are indicated.

Fig. 4 Phenotypic traits of the evolved clones sampled from each replicate population both in their respective evolution environment $(O)$ and in the three alternative ones $(\boldsymbol{O})$. The $\mu_{\max }(\mathrm{a}$ to d) and fitness (e to h) values (+/- SEM) of each evolved clone are given in each of the Ace, Gly, Glc, and Glu environments (indicated from left to right on the x-axes). The evolution environment from which each set of four clones was sampled is indicated at the top of each panel: Ace (a, e), Gly (b, f), Glc (c, g), and Glu (d, h). Note that y-axis scales vary.

Fig. 5 Among-clone phenotypic variability in the alternative environments. Each small hollow symbol (O) corresponds to a comparison between a pair of clones in one of the alternative environments. The y-axis shows the phenotypic variability (mean squares) ranked within each alternative environment, a high rank value indicating that the pair of clones displays very different phenotypes (due to tied ranks, not all of them are seen on the figure). Phenotypic variability is given for $\mu_{\max }(\mathrm{a}, \mathrm{c})$ and fitness $(\mathrm{b}, \mathrm{d})$ values for pairs of clones that evolved in different (a, b), and identical (c, d) environments. Median ranks ( $)$ as well as first and third

675 Table 1 Mutations identified in the 16 evolved clones sampled after 1000 generations of 676 evolution in four different environments. 


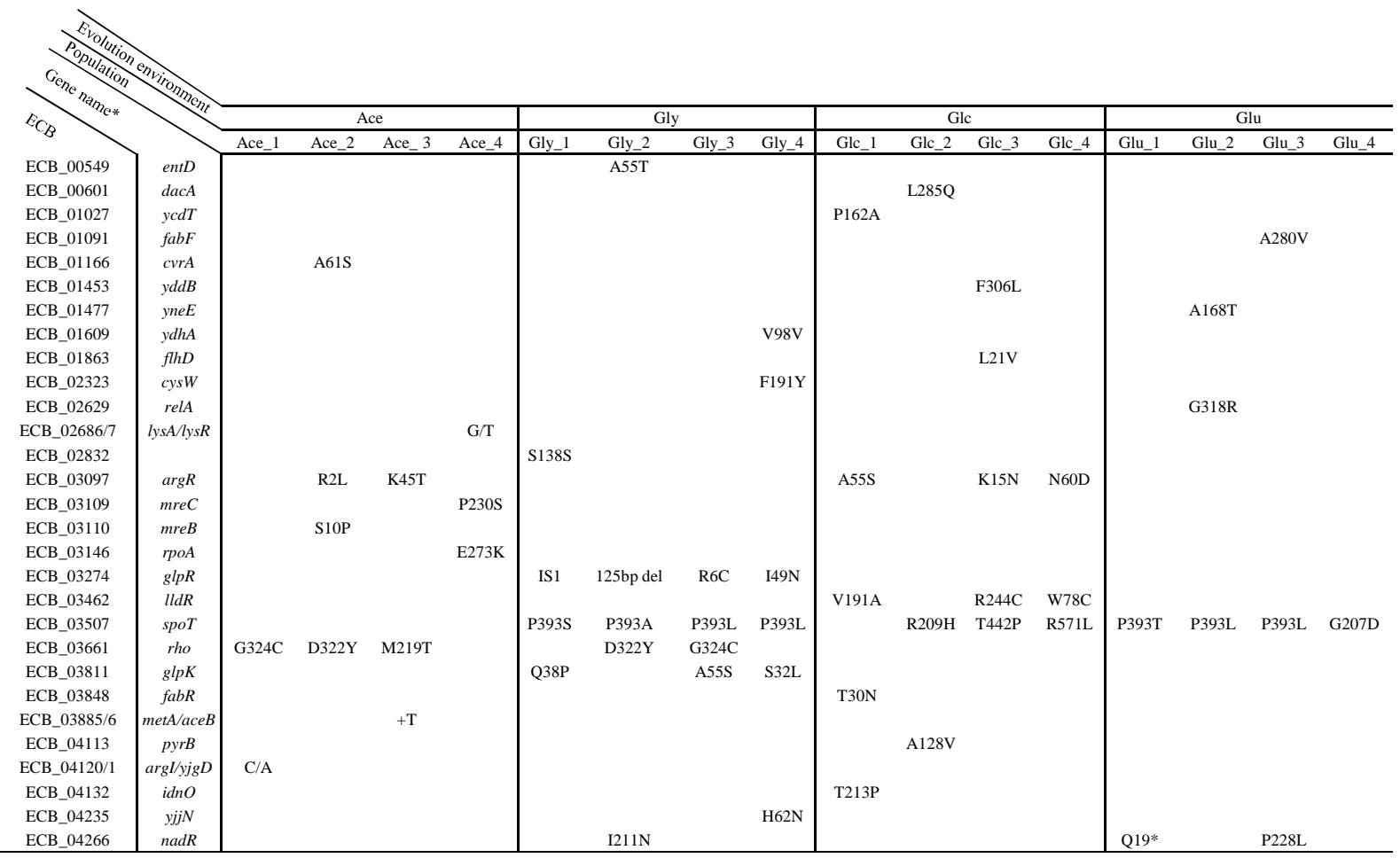

$678 *$ The name of the genes carrying mutations are given, together with their ECB numbers

679 (Jeong et al. 2009). For intergenic mutations, the name of the two surrounding genes is given.

681 Table 2 Variability of the correlated phenotypic responses for each pair of clones in the

682 alternative environments as a function of genetic parallelism (shared mutated genes or not)

683 and evolutionary history (same evolution environment or not). Likelihood ratio $\chi^{2}$ tests on

684 ordinal logistic regressions (GLM, Material and methods) were performed with the among-

685 clone variability (Mean square among clones) of the $\mu_{\max }$ and fitness values (ranked within

686 each alternative environment) taken as ordinal response variables. Evolution in identical

687 environments and level of genetic parallelism among clones were taken as explanatory

688 variables.

\begin{tabular}{lccc}
\hline Source & DF & $\chi^{2}$ & $P$-value \\
\hline$\mu_{\max }$ & & & \\
Whole model $^{*}$ & 3 & 73.07 & $<0.0001$ \\
Evolutionary history & 1 & 33.88 & $<0.0001$
\end{tabular}


Genetic parallelism

Environment X Parallelism

$\begin{array}{rrr}1 & 3.55 & 0.0596 \\ 1 & 14.61 & 0.0001\end{array}$

Fitness

Whole model ${ }^{*}$

$3 \quad 24.56 \quad<0.0001$

Evolutionary history

$\begin{array}{lll}1 & 14.60 & 0.0001\end{array}$

Genetic parallelism

$\begin{array}{lll}1 & 0.08 & 0.7809\end{array}$

Environment X Parallelism

$\begin{array}{lll}1 & 4.60 & 0.0320\end{array}$

690 "Indicates whether the model based on the two explanatory variables as well as their

691 interaction significantly explained the among clone variability.

692

693

694 
(a)

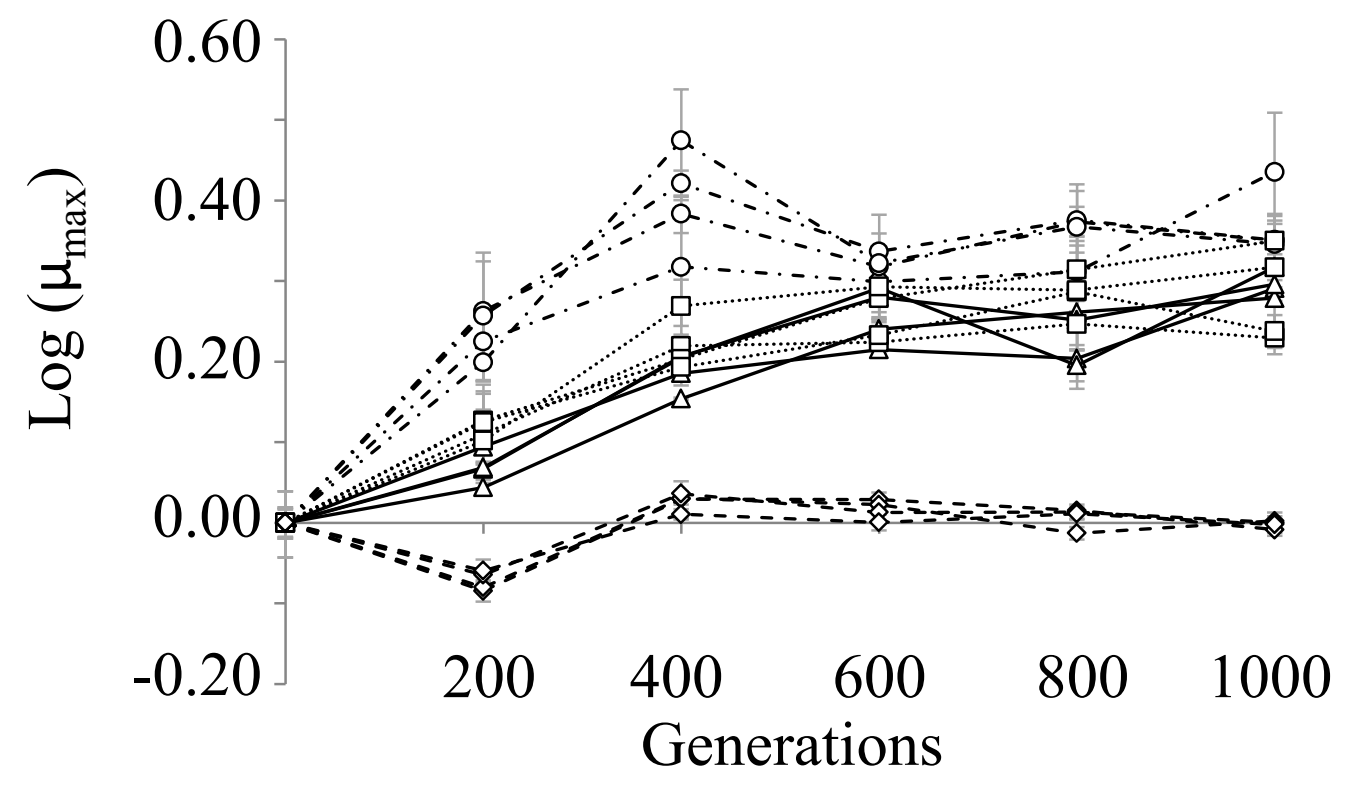

(b)

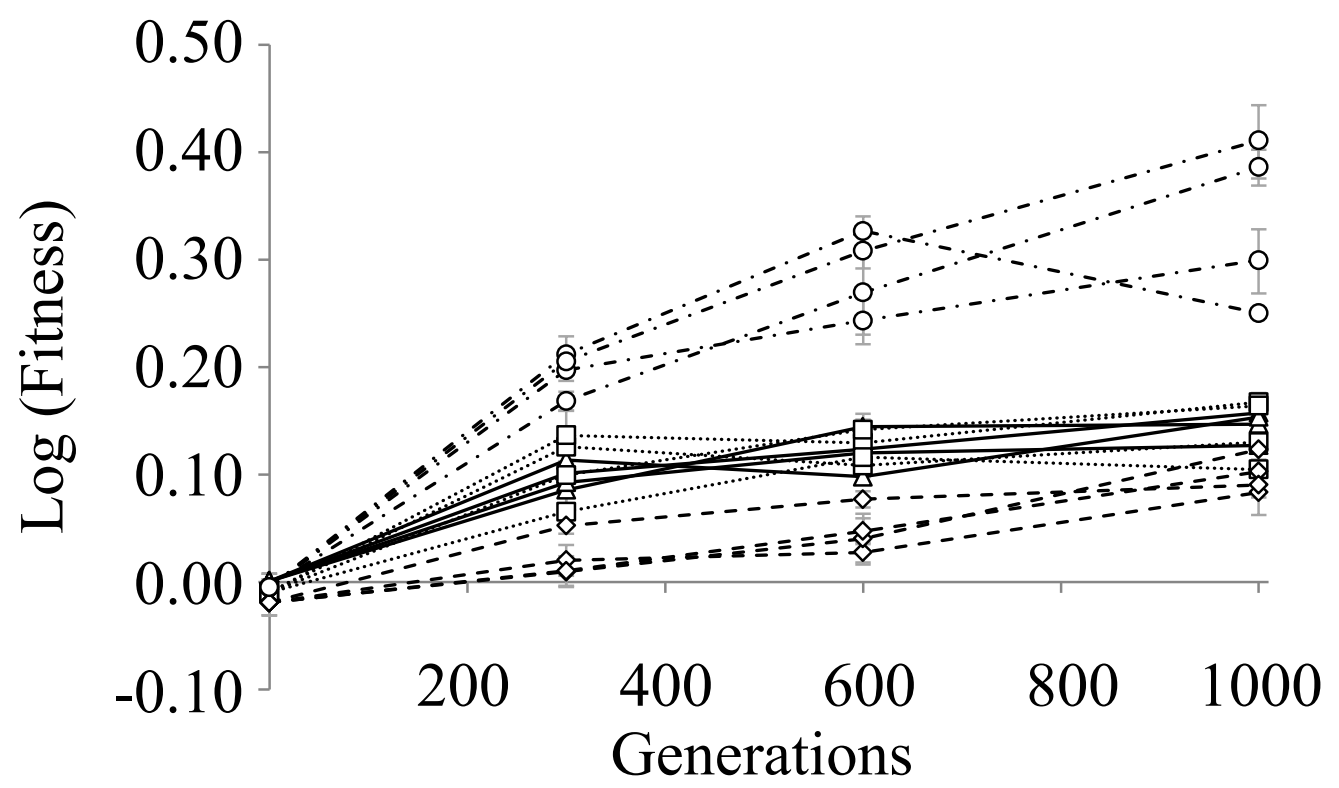




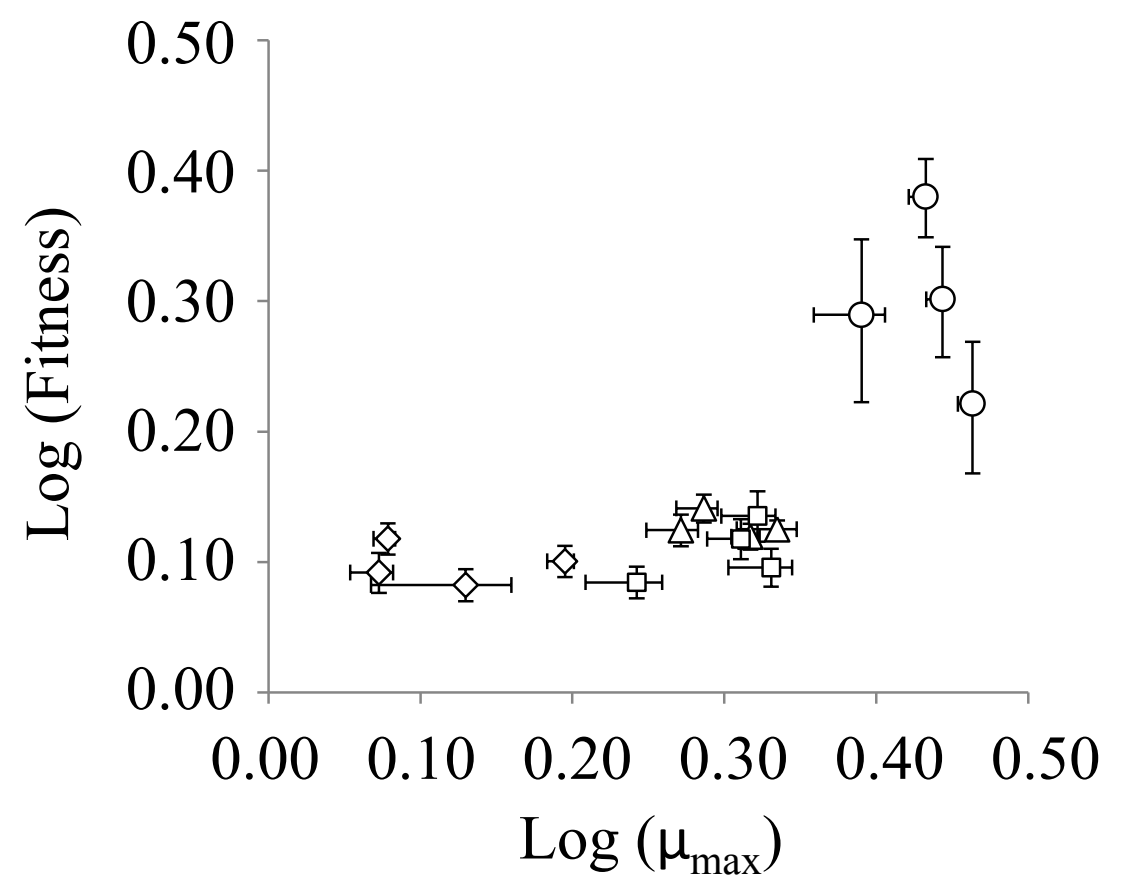




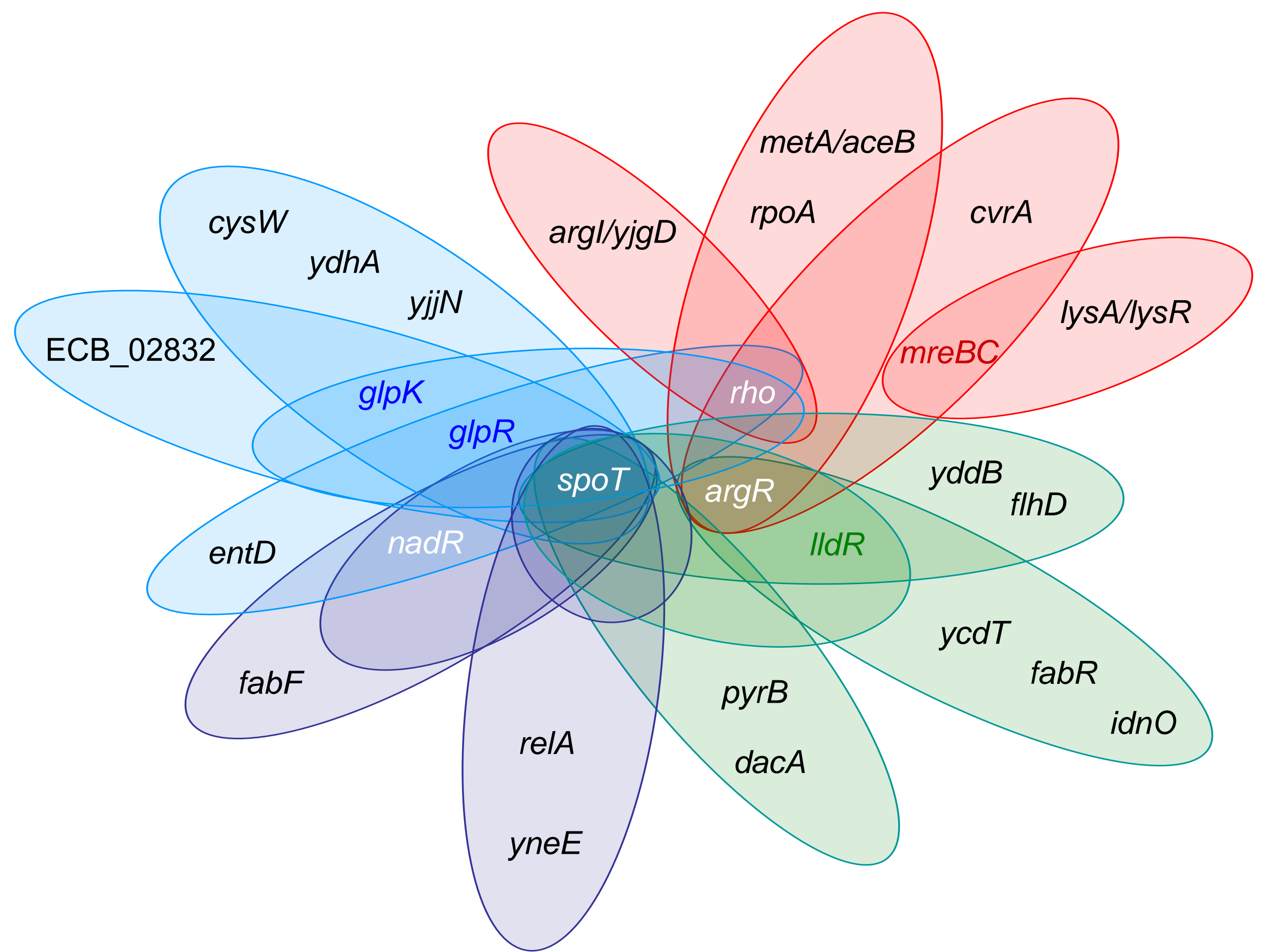


(a)

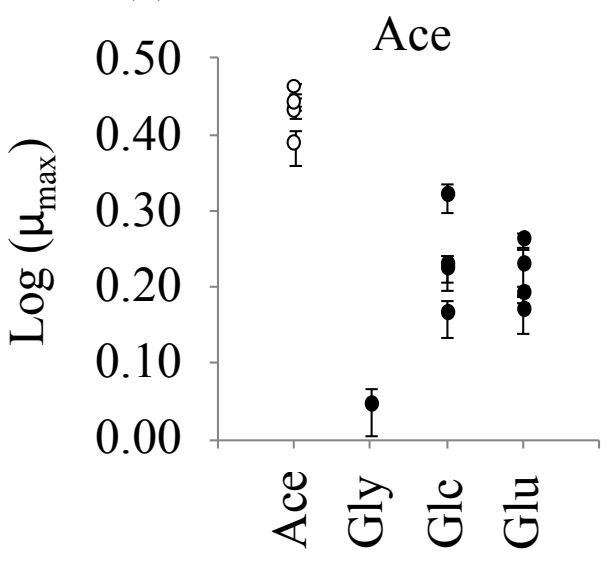

(b)

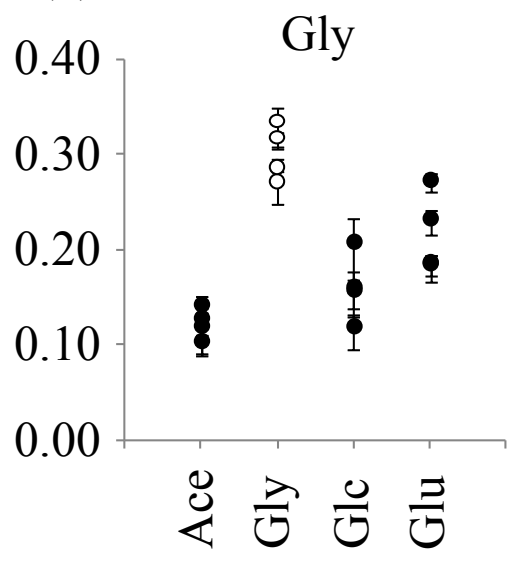

(c)

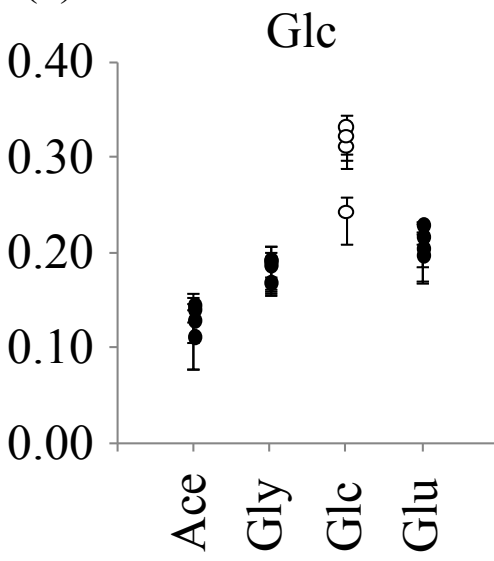

(d)

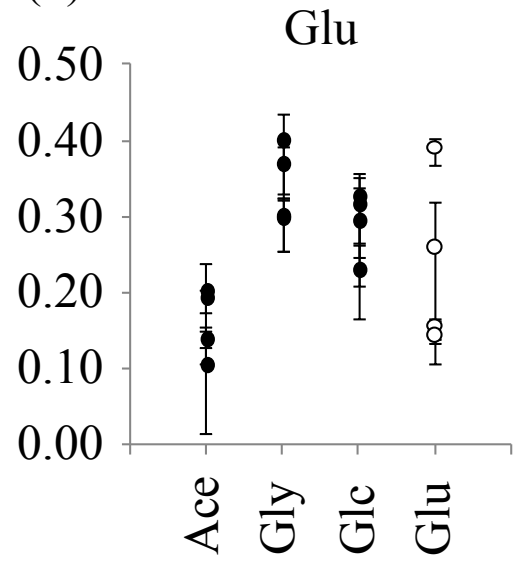

Environment

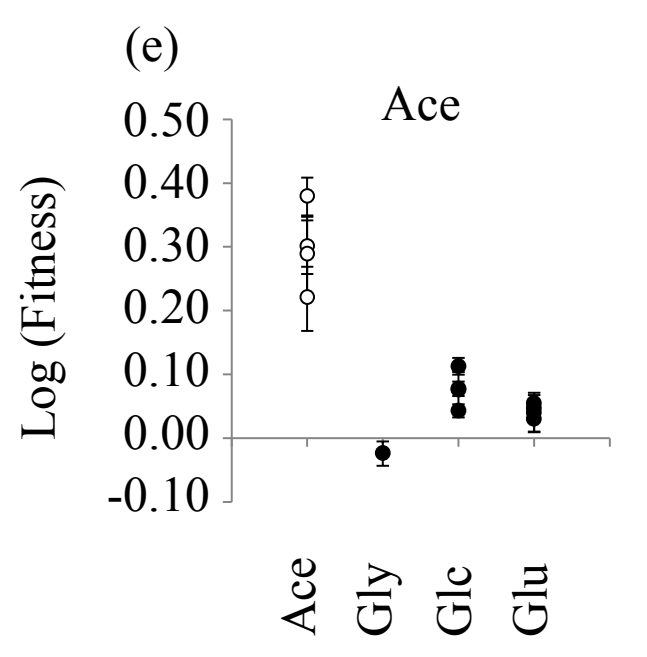

(f)

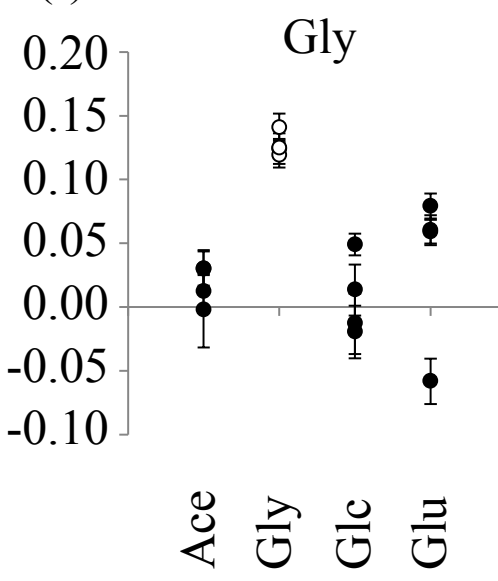

(g)

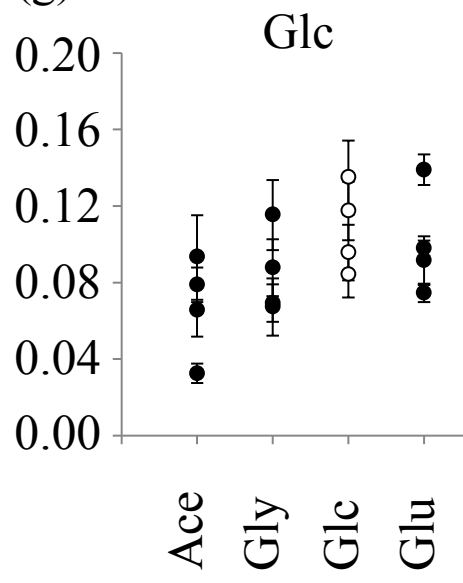

(h)

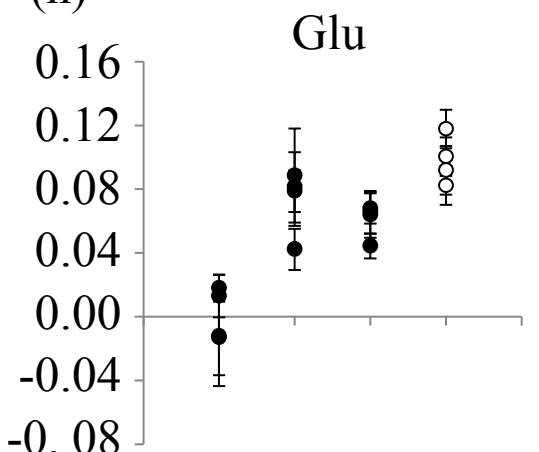

Environment 
(a)

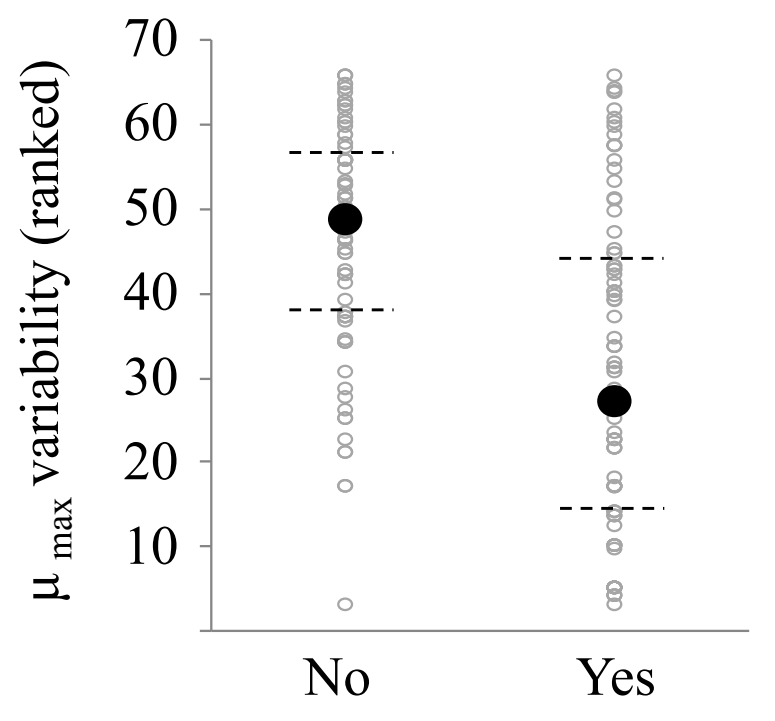

(b)

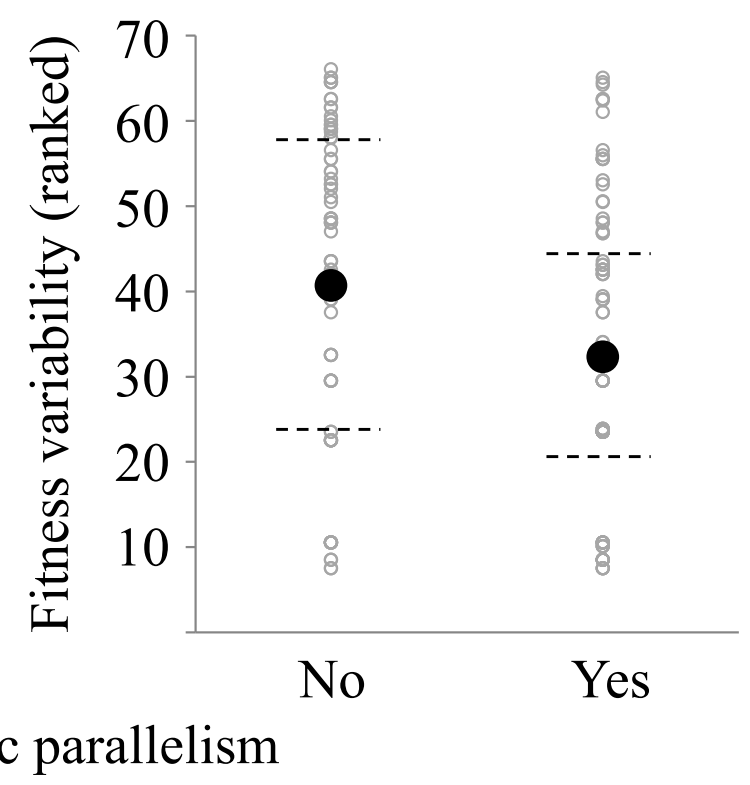

(c)

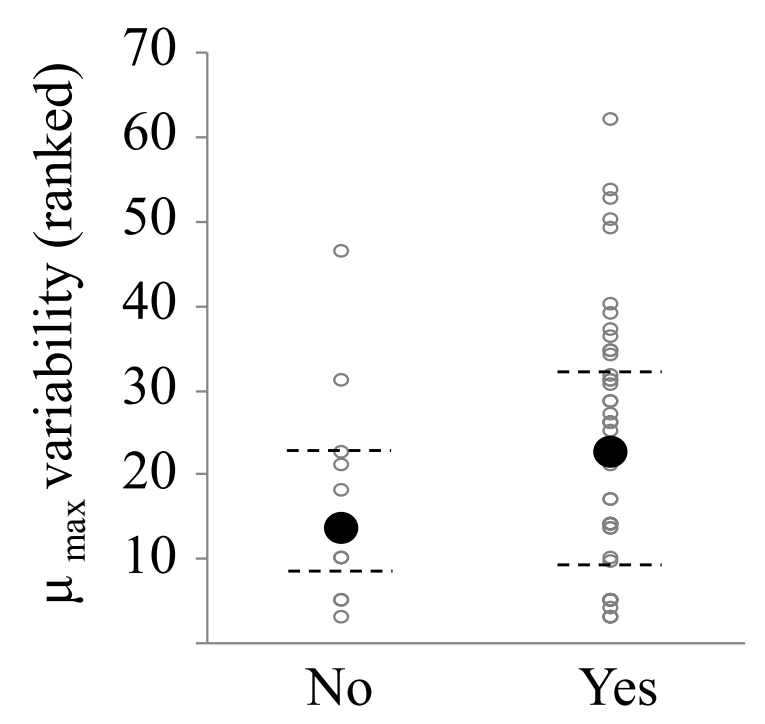

(d)

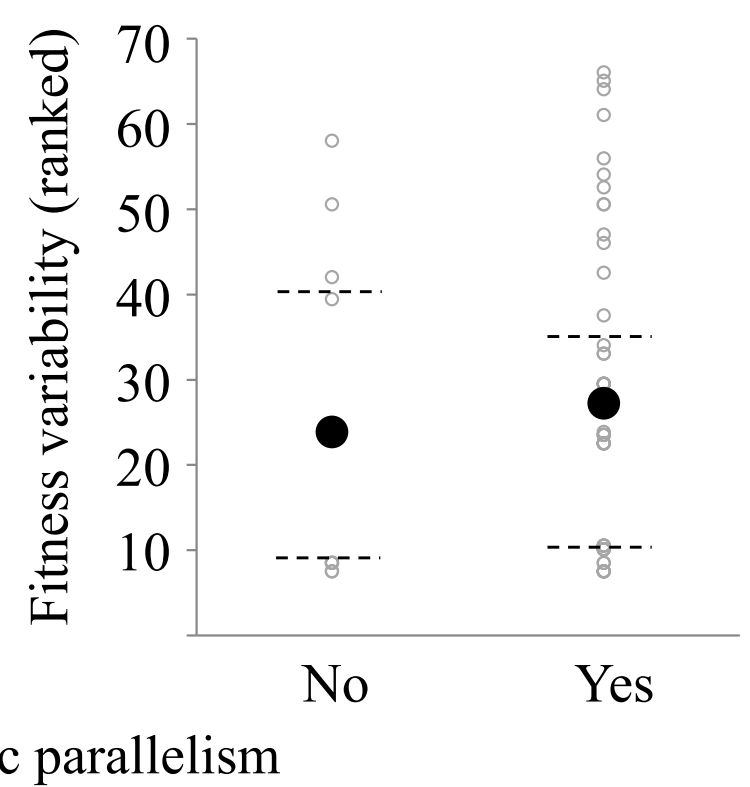


Supporting information

\section{Evolutionary history and genetic parallelism affect correlated responses to evolution}

Mickael Le Gac, Tim Cooper, Stéphane Cruveiller, Claudine Médigue, and Dominique

Schneider

This file includes:

Table S1 Phenotypic traits ( $\mu_{\max }$ and fitness) of the 16 populations during evolutionary time in their respective evolution environments.

Table S2 Phenotypic traits ( $\mu_{\max }$ and fitness) of the evolved clones sampled from each of the 16 populations in the four environments.

Table S3 Among-clone coefficient of variation in the four environments for the two phenotypic traits $\mu_{\max }$ and fitness. 
Table S1 Phenotypic traits ( $\mu_{\max }$ and fitness) of the 16 populations during evolutionary time in their respective evolution environments

\begin{tabular}{|c|c|c|c|c|c|c|c|c|c|c|}
\hline \multirow{2}{*}{ Population } & & \multicolumn{5}{|c|}{$\mu_{\max } *$} & \multicolumn{4}{|c|}{ fitness* } \\
\hline & 0 & 200 & 400 & 600 & 800 & 1000 & 0 & 300 & 600 & 1000 \\
\hline Ace 1 & & $1.68+/-0.10$ & $2.08+/-0.32$ & $1.99+/-0.09$ & $2.05+/-0.22$ & $2.73+/-0.50$ & & $2.11+/-0.10$ & $1.77+/-0.06$ & $1.39+/-0.05$ \\
\hline Ace 2 & & $1.83+/-0.33$ & $2.42+/-0.13$ & $2.08+/-0.21$ & $2.36+/-0.10$ & $2.24+/-0.18$ & & $1.94+/-0.05$ & $1.87+/-0.19$ & $2.40+/-0.22$ \\
\hline Ace 3 & $1.00+--0.09$ & $1.81+/-0.30$ & $2.64+/-0.10$ & $2.17+/-0.02$ & $2.37+/-0.21$ & $2.24+/-0.13$ & $0.97+/-0.06$ & $2.28+/-0.14$ & $1.73+/-0.14$ & $1.41+/-0.03$ \\
\hline Ace 4 & & $1.58+/-0.08$ & $2.98+/-0.47$ & $2.10+/-0.31$ & $2.33+/-0.30$ & $2.22+/-0.18$ & & $1.79+/-0.11$ & $1.48+/-0.08$ & $1.38+/-0.07$ \\
\hline Gly_1 & & $1.24+/-0.01$ & $1.53+/-0.08$ & $1.64+/-0.07$ & $1.60+/-0.06$ & $1.95+/-0.21$ & & $1.55+/-0.10$ & $1.95+/-0.15$ & $2.15+/-0.10$ \\
\hline Gly 2 & & $1.11+/-0.02$ & $1.43+/-0.01$ & $1.74+/-0.06$ & $1.83+/-0.18$ & $1.90+/-0.13$ & & $1.47+/-0.08$ & $1.71+/-0.05$ & $1.81+/-0.06$ \\
\hline Gly 3 & .02 & $1.17+/-0.02$ & $1.61+/-0.05$ & $1.91+/-0.03$ & $1.79+/-0.15$ & $1.98+/-0.09$ & $1.00+/-0.05$ & $1.51+/-0.05$ & $1.84+/-0.05$ & $1.68+/-0.10$ \\
\hline Gly_4 & & $1.17+/-0.01$ & $1.60+/-0.01$ & $1.95+/-0.20$ & $1.57+/-0.07$ & $2.08+/-0.08$ & & $1.56+/-0.09$ & $1.61+/-0.09$ & $1.94+/-0.22$ \\
\hline Glc_1 & & $1.29+/-0.17$ & $1.66+/-0.04$ & $1.68+/-0.05$ & $1.76+/-0.02$ & $1.70+/-0.08$ & & $1.63+/-0.12$ & $1.56+/-0.09$ & $1.74+/-0.14$ \\
\hline Glc_2 & & $1.34+/-0.04$ & $1.60+/-0.08$ & $1.90+/-0.11$ & $2.06+/-0.18$ & $2.24+/-0.11$ & & $1.58+/-0.12$ & $1.69+/-0.05$ & $2.12+/-0.17$ \\
\hline Glc 3 & .04 & $1.33+/-0.15$ & $1.56+/-0.04$ & $1.71+/-0.07$ & $1.94+/-0.27$ & $1.73+/-0.08$ & 3 & $1.52+/-0.06$ & $1.78+/-0.14$ & $2.12+/-0.00$ \\
\hline Glc-4 & & $1.26+/-0.18$ & $1.86+/-0.15$ & $1.96+/-0.14$ & $1.94+/-0.16$ & $2.08+/-0.12$ & & $1.32+/-0.05$ & $1.50+/-0.06$ & $1.53+/-0.05$ \\
\hline Glu_1 1 & & $0.82+/-0.03$ & $1.07+/-0.06$ & $1.07+/-0.02$ & $1.04+/-0.01$ & $0.98+/-0.02$ & & $1.14+/-0.12$ & $1.13+/-0.04$ & $1.39+/-0.14$ \\
\hline Glu_2 & & $0.83+/-0.01$ & $1.07+/-0.02$ & $1.05+/-0.01$ & $0.97+/-0.02$ & $1.01+/-0.01$ & & $1.03+/-0.06$ & $1.21+/-0.13$ & $1.41+/-0.07$ \\
\hline Glu_3 3 & $+/-0.04$ & $0.86+/-0.00$ & $1.09+/-0.01$ & $1.03+/-0.00$ & $1.03+/-0.02$ & $1.00+/-0.03$ & $0.91+/-0.06$ & $1.06+/-0.07$ & $1.21+/-0.03$ & $1.39+/-0.08$ \\
\hline Glu_4 & & $0.87+/-0.03$ & $1.02+/-0.02$ & $1.00+/-0.02$ & $1.03+/-0.01$ & $1.00+/-0.01$ & & $1.28+/-0.03$ & $1.45+/-0.08$ & $1.52+/-0.02$ \\
\hline
\end{tabular}

${ }^{*} \mu_{\max }$ and fitness values are presented as log-transformed in Figure 1 but not in Table S1. 
Table S2 Phenotypic traits ( $\mu_{\max }$ and fitness) of the evolved clones sampled from each of the 16 populations in the four environments

\begin{tabular}{|c|c|c|c|}
\hline Environnent & Population & $\mu_{\max }{ }^{*}$ & fitness ${ }^{*}$ \\
\hline \multirow{16}{*}{ Ace } & Ace_1 & $2.71+/-0.07$ & $1.36+/-0.06$ \\
\hline & Ace_2 & $2.78+/-0.07$ & $1.27+/-0.04$ \\
\hline & Ace 33 & $2.46+/-0.17$ & $1.31+/-0.06$ \\
\hline & Ace 4 & $2.91+/-0.06$ & $1.34+/-0.08$ \\
\hline & Gly_1 & Very slow growth ${ }^{* *}$ & Very slow growth \\
\hline & Gly_2 & Very slow growth & Very slow growth \\
\hline & Gly_3 & Very slow growth & Very slow growth \\
\hline & Gly 4 & $1.12+/-0.11$ & $0.90+/-0.07$ \\
\hline & Glc_1 & $2.10+/-0.12$ & $1.22+/-0.02$ \\
\hline & Glc_2 & $1.70+/-0.09$ & $1.17+/-0.07$ \\
\hline & Glc_3 & $1.68+/-0.11$ & $1.22+/-0.03$ \\
\hline & $\mathrm{Glc}_{-}^{-} 4$ & $1.47+/-0.11$ & $1.15+/-0.05$ \\
\hline & Glu_1 & $1.56+/-0.05$ & $1.11+/-0.08$ \\
\hline & Glu_2 & $1.70+/-0.16$ & $1.06+/-0.04$ \\
\hline & Glu_3 & $1.49+/-0.11$ & $1.14+/-0.04$ \\
\hline & Glu_4 & $1.84+/-0.06$ & $1.10+/-0.04$ \\
\hline \multirow{16}{*}{ Gly } & Ace_1 & $1.39+/-0.06$ & $1.19+/-0.13$ \\
\hline & Ace_2 & $1.27+/-0.04$ & $1.19+/-0.10$ \\
\hline & Ace 3 & $1.34+/-0.06$ & $1.05+/-0.14$ \\
\hline & Ace 4 & $1.32+/-0.08$ & $1.03+/-0.05$ \\
\hline & Gly_1 & $1.87+/-0.10$ & $2.09+/-0.26$ \\
\hline & Gly_2 & $1.93+/-0.08$ & $1.97+/-0.12$ \\
\hline & Gly_3 & $2.08+/-0.06$ & $1.61+/-0.06$ \\
\hline & Gly_4 & $2.16+/-0.13$ & $1.94+/-0.08$ \\
\hline & Glc_1 & $1.45+/-0.10$ & $0.98+/-0.06$ \\
\hline & Glc_2 & $1.61+/-0.19$ & $1.00+/-0.05$ \\
\hline & Glc_3 & $1.44+/-0.06$ & $1.09+/-0.06$ \\
\hline & Glc_4 & $1.32+/-0.07$ & $1.28+/-0.08$ \\
\hline & Glu_1 & $1.71+/-0.07$ & $1.37+/-0.13$ \\
\hline & Glu_2 & $1.54+/-0.05$ & $0.80+/-0.05$ \\
\hline & Glu_3 & $1.87+/-0.05$ & $1.43+/-0.10$ \\
\hline & Glu_4 & $1.53+/-0.06$ & $1.26+/-0.07$ \\
\hline \multirow{16}{*}{ Glc } & Ace_1 & $1.29+/-0.10$ & $1.34+/-0.07$ \\
\hline & Ace_2 & $1.34+/-0.15$ & $1.54+/-0.12$ \\
\hline & Ace_3 & $1.39+/-0.02$ & $1.39+/-0.05$ \\
\hline & Ace $^{-} 4$ & $1.38+/-0.11$ & $1.25+/-0.05$ \\
\hline & Gly_1 & $1.56+/-0.10$ & $1.42+/-0.07$ \\
\hline & Gly_2 & $1.54+/-0.09$ & $1.79+/-0.10$ \\
\hline & Gly_3 & $1.47+/-0.04$ & $1.42+/-0.10$ \\
\hline & Gly_4 & $1.55+/-0.11$ & $1.40+/-0.07$ \\
\hline & Glc_1 & $2.05+/-0.10$ & $1.62+/-0.11$ \\
\hline & Glc_2 & $2.14+/-0.14$ & $1.51+/-0.09$ \\
\hline & Glc_3 & $1.75+/-0.13$ & $1.39+/-0.06$ \\
\hline & $\mathrm{Glc}^{-} 4$ & $2.10+/-0.11$ & $1.54+/-0.06$ \\
\hline & Glu_1 & $1.64+/-0.11$ & $1.53+/-0.04$ \\
\hline & Glu_2 & $1.60+/-0.13$ & $1.43+/-0.04$ \\
\hline & Glu_3 & $1.69+/-0.04$ & $1.63+/-0.04$ \\
\hline & Glu_4 & $1.57+/-0.09$ & $1.32+/-0.02$ \\
\hline \multirow{6}{*}{ Glu } & Ace_1 & $1.16+/-0.11$ & $0.95+/-0.17$ \\
\hline & Ace 2 & $1.25+/-0.03$ & $1.10+/-0.09$ \\
\hline & Ace 33 & $1.26+/-0.10$ & $1.09+/-0.05$ \\
\hline & Ace 4 & $1.17+/-0.04$ & $0.97+/-0.19$ \\
\hline & Gly_1 & $1.41+/-0.07$ & $2.14+/-0.50$ \\
\hline & Gly_2 & $1.53+/-0.08$ & $1.55+/-0.17$ \\
\hline
\end{tabular}




\begin{tabular}{lll} 
Gly_3 & $1.59+/-0.13$ & $1.86+/-0.24$ \\
Gly_4 & $1.42+/-0.07$ & $1.86+/-0.18$ \\
\hline Glc_1 & $1.30+/-0.10$ & $1.45+/-0.14$ \\
Glc_2 & $1.41+/-0.14$ & $1.44+/-0.07$ \\
Glc_3 & $1.44+/-0.12$ & $1.46+/-0.13$ \\
Glc_4 & $1.46+/-0.10$ & $1.30+/-0.05$ \\
\hline Glu_1 & $1.20+/-0.03$ & $2.28+/-0.15$ \\
Glu_2 & $1.57+/-0.04$ & $2.12+/-0.20$ \\
Glu_3 & $1.35+/-0.19$ & $2.00+/-0.19$ \\
Glu_4 & $1.18+/-0.05$ & $2.00+/-0.27$
\end{tabular}

The $\mu_{\max }$ and fitness values are given (+/-SEM) for each evolved clone sampled from each of the 16 evolving populations in each of the four different environments.

${ }^{*} \mu_{\max }$ values are presented as log-transformed in Figure 2 but not in Table S2.

${ }^{* *}$ As no quantitative values could be obtained for these measures, they were not included in the statistical analyses. 
Table S3 Among-clone coefficient of variation in the four environments for the two phenotypic traits $\mu_{\max }$ and fitness

\begin{tabular}{cccc}
\hline \multirow{2}{*}{ Evolution environment } & Alternative & \multicolumn{2}{c}{ Coefficient of variation } \\
\cline { 2 - 4 } & environment & $\mu_{\max }$ & fitness \\
\cline { 2 - 4 } Ace & Ace & 0.07 & 0.15 \\
& Gly & 0.04 & 0.04 \\
& Glc & 0.03 & 0.06 \\
& Glu & 0.05 & 0.04 \\
\hline \multirow{3}{*}{ Gly } & Ace & Very slow growth \\
& Gly & 0.07 & 0.02 \\
& Glc & 0.03 & 0.05 \\
& Glu & 0.06 & 0.05 \\
\hline \multirow{3}{*}{ Glc } & Ace & 0.15 & 0.07 \\
& Gly & 0.08 & 0.07 \\
& Glc & 0.09 & 0.05 \\
& Glu & 0.05 & 0.02 \\
\hline \multirow{3}{*}{ Glu } & Ace & 0.09 & 0.02 \\
& Gly & 0.10 & 0.13 \\
& Glc & 0.03 & 0.06 \\
& Glu & 0.13 & 0.03 \\
\hline
\end{tabular}

${ }^{*}$ As no quantitative values could be obtained for these measures, they were not included in the statistical analyses. 